\title{
Outcome of treatment of multidrug resistant tuberculosis
}

\author{
WV Senaratne ${ }^{1}$
}

\begin{abstract}
Multidrug resistant tuberculosis (MDRTB) is a growing problem worldwide. It is an emerging problem in Sri Lanka too although the exact data are not known. This report describes treatment and outcome of 14 MDRTB patients. All have had previous anti-tuberculosis treatment. Out of 32 previous treatment episodes, treatment has been either irregular or defaulted in $26(81 \%)$, which has largely contributed to the emergence of drug resistance.
\end{abstract}

Treatment commenced with a combination of second line anti-tuberculosis drugs, namely, amikacin, ciprofloxacin, ethionamide, clofazimine and pyrazinamide. Four patients were considered cured, another four failed treatment, five defaulted treatment and one died. Previous sensitivity to second line drugs and proper isolation facilities were not available.

Preparedness to face the threat of MDRTB is essential. Measures should be taken to reduce the rate of defaulters of anti-tuberculosis treatment, and facilities to treat MDRTB should be expanded.

\section{Introduction}

Tuberculous infection with organisms resistant to both rifampicin and isoniazid, with or without resistance to other anti-tuberculosis drugs, is defined as MDRTB. It is a growing problem globally [1]. In Sri Lanka also it is an emerging problem. The Central Laboratory of the tuberculosis control programme has identified 12 and 7 MDR isolates in 2001 and 2002, respectively. In most cases MDRTB results from erratic treatment.

\section{Material}

Fourteen patients with MDRTB, diagnosed between November 1997 to August 2002, were analysed. Sputum cultures were done in Lowenstein-Jensen medium and sensitivity to rifampicin, isoniazid, ethambutol and streptomycin was done by the dilution method. None of the patients had received second line drugs previously.

\section{Results}

There were three women and 11 men. Their ages ranged from 24 to 84 years (mean 50). None of the patients were new, and none had contact with a known drug resistant tuberculosis patient. Treatment in chest clinics consisted of Category 1 for the first episode and Category 2 for subsequent episodes. Only one patient had supervised treatment during the intensive phase of Category 1 treatment. However, she had vomiting on some days after taking the treatment.

Intensive phase of Category 2 treatment has been supervised for varying periods. None of the patients had continuation of treatment supervised. Out of a total of 32 treatment episodes, six have been considered as either cured or treatment completed. Patients have either defaulted or taken irregular treatment during other episodes.

Chest xray showed bilateral involvement in eight, one lung involvement in three and one zonal involvement in three patients.

Sensitivity tests (Table 1) showed Mycobacterium tuberculosis (MTB) resistant to both rifampicin and isoniazid in all cases, resistance to streptomycin in nine and resistance to ethambutol in 10 . Sensitivity to pyrazinamide was not done.

Treatment consisted of ciprofloxacin, ethionamide and clofazimine, supplemented by amikacin and pyrazinamide during the intensive phase (3-4 months). Where there was sensitivity of the MTB to ethambutol and streptomycin, they too were added.

Three patients became sputum direct smear and culture negative at the end of the intensive phase of treatment, and remained so for more than 18 months of the continuation phase. One patient who was sputum positive after 4 months of intensive phase treatment became sputum negative during the continuation phase. At the time of writing this article, she remains sputum direct smear negative and culture negative for 10 months. These four patients are considered cured. One patient who was negative at the end of the intensive phase became positive after 8 months of treatment. Inclusive of this patient, the total number of treatment failures in this series was four. Three patients refused treatment from the onset and were considered as defaulters. Two other patients defaulted treatment after 2 to 3 months, making total number defaulted as five. One died during treatment.

\section{Discussion}

It is recommended that treatment for MDRTB is commenced with at least three, but preferably as many as six or seven drugs, to which the MTB is likely to be sensitive $[2,3]$. It should include an aminoglycoside and a quinolone. Aminoglycoside can be either streptomycin (if organisms are sensitive) or amikacin/kanamycin. Ethambutol is added if the organisms are sensitive to it and pyrazinamide is always included in a re-treatment

${ }^{1}$ Chest Physician, Chest Hospital, Welisara, Ragama, Sri Lanka. Tel: +94 112958271, +94 2956702, e-mail: <wichitra@dialogsl.net> (Competing interests: none declared). Received 22 July 2003 and revised version accepted 2 December 2003. 
Table 1. Treatment details and outcome in 14 patients with MDRTB

\begin{tabular}{|c|c|c|c|}
\hline & Sensitivity & Treatment & Outcome \\
\hline 1. & $\begin{array}{l}\text { Resistant to } \mathrm{R}, \mathrm{H} \\
\text { Sensitive to } \mathrm{E}, \mathrm{S}\end{array}$ & E, S, Z, followed by E daily and S three days a week. & Cured \\
\hline 2. & Resistant to R, H, S, E & Am, Ci, Etm, Z, Cl, followed by $\mathrm{Ci}$, Etm & Cured \\
\hline 3. & $\begin{array}{l}\text { Resistant to R, H, E } \\
\text { Sensitive to } \mathrm{S}\end{array}$ & Refused treatment & Defaulter \\
\hline 4. & Resistant to R, H, S, E & $\mathrm{Am}, \mathrm{Ci}, \mathrm{Etm}, \mathrm{Z}, \mathrm{Cl}$ & Defaulter \\
\hline 5. & Resistant to R, H, S, E & Refused indoor treatment. Outdoor $\mathrm{Ci}, \mathrm{Etm}, \mathrm{Z}, \mathrm{Cl}$ & Treatment failure \\
\hline 6. & Resistant to R, H, S, E & Refused treatment & Defaulter \\
\hline 7. & Resistant to R, H, S, E & Am, Ci, Etm. Z, Cl, followed by $\mathrm{Ci}, \mathrm{Etm}$ and $\mathrm{Cl}$ & Treatment failure \\
\hline 8. & $\begin{array}{l}\text { Resistant to R, H, S } \\
\text { Sensitive to E }\end{array}$ & $\begin{array}{l}\mathrm{Am}, \mathrm{Ci}, \mathrm{E}, \mathrm{Cl} \text {, followed by E, } \mathrm{Ci} \\
\text { Patient could not tolerate } \mathrm{Z}, \mathrm{Etm}\end{array}$ & Cured \\
\hline 9. & $\begin{array}{l}\text { Resistant to R, H } \\
\text { Sensitive to E, S }\end{array}$ & $\mathrm{S}, \mathrm{Ci}, \mathrm{Etm}, \mathrm{E}, \mathrm{Z}, \mathrm{Cl}$ & Defaulter \\
\hline 10. & $\begin{array}{l}\text { Resistant to R, H } \\
\text { Sensitive to E, } S\end{array}$ & Am. Ci. Z, E, Etm, Cl, followed by Ci, E, Etm & $\begin{array}{l}\text { Treatment } \\
\text { failure }\end{array}$ \\
\hline 11. & $\begin{array}{l}\text { Resistant to R, H, E } \\
\text { Sensitive to } \mathrm{S}\end{array}$ & Am, $\mathrm{Ci}, \mathrm{Z}, \mathrm{Etm}, \mathrm{Cl}$, followed by $\mathrm{Ci}, \mathrm{Etm}, \mathrm{Cl}$ & $\begin{array}{l}\text { Treatment } \\
\text { failure }\end{array}$ \\
\hline 12. & Resistant to R, H, S, E & Am, $\mathrm{Ci}, \mathrm{Z}, \mathrm{Etm}, \mathrm{Cl}$, followed by $\mathrm{Ci}, \mathrm{Etm}, \mathrm{Cl}$ & Cured \\
\hline 13. & Resistant to R, H, S, E & Refused treatment & Defaulter \\
\hline 14. & Resistant to R, H, S, E & $\mathrm{Am}, \mathrm{Ci}, \mathrm{Z}, \mathrm{Etm}, \mathrm{Cl}$ & Died \\
\hline
\end{tabular}

Am-amikacin, Ci-ciprofloxacin, Cl-clofazimine, E-ethambutol, Etm-ethionamide, H-isoniazid, R-rifampicin, S-streptomycin, Z-pyrazinamide.

regimen, as resistance to pyrazinamide is neither easy to acquire nor proved by susceptibility tests [3]. The second line anti-tuberculosis drugs which our patients received were amikacin, ciprofloxacin, ethionamide and clofazimine. Due to unavailability of facilities for pretreatment sensitivity tests to second line drugs, these were not done. In this series, only four out of 14 could be considered cured. The success rate of MDRTB treatment is low. However, several studies done with carefully selected treatment regimens have shown favourable outcome, clinically and microbiologically $[4,5]$. When compared to these studies, the present series shows a low success rate. This could have been due to many factors. Firstly, second line anti-tuberculosis drugs such as cycloserine were not included as they are not available in Sri Lanka. Secondly, efficacy of clofazimine in MDRTB is doubtful but was still used for want of a second line drug in the absence of better alternatives. Thirdly, although the quinolone which our patients received was ciprofloxacin, ofloxacin is shown to be more effective in the treatment of MDRTB because of better pharmacokinetic properties [3]. During the intensive phase of treatment patients were kept in the usual TB wards as there was no proper isolation facility. This carries a risk of spreading MDRTB to other patients as well as the staff. Under ideal conditions, patients should be isolated in negatively ventilated rooms, vented out with facilities to filter air through high efficiency particulate air (HEPA) filters and ultraviolet germicidal irradiation (UVGI).

\section{Conclusions}

A national policy on the management of MDRTB patients needs to be formulated. Such a policy should address the key issues such as: (i) making available more of second line anti-tuberculosis drugs for exclusive use in the centres which manage MDRTB patients under the supervision of chest physicians, (ii) providing laboratory facilities to do sensitivity tests to second line drugs, and (iii) providing isolation facilities to minimise spread of the disease and taking measures to protect the health personnel.

\section{References}

1. Espinal MA, Laszlo A, Simonsen L, Boulahbal F, Kim S $\mathrm{J}$, et al. Global trends in resistance to anti-tuberculosis drugs. New England Journal of Medicine 2001; 344: 1294-303.

2. Iseman MD. Treatment of multidrug resistant tuberculosis. New England Journal of Medicine 1993; 329: 784-91.

3. Guidelines for management of drug resistant tuberculosis. WHO/ TB/96.210 (Rev.1), Geneva, 1997.

4. Park SK, Kim CT, Song SD. Outcome of chemotherapy in 107 patients with pulmonary tuberculosis resistant to isoniazid and rifampicin. International Journal of Tuberculosis and Lung Disease 1995; 2: 877-84.

5. Goble M, Iseman MD, Madsen LA, Waite D, Ackerson $\mathrm{L}$, et al. Treatment of 171 patients with pulmonary tuberculosis resistant to isoniazid and rifampicin. New England Journal of Medicine 1993; 328: 527-32. 